\title{
Effects of Edaravone on Functional Recovery of a Rat Model with Spinal Cord Injury Through Induced Differentiation of Bone Marrow Mesenchymal Stem Cells into Neuron-Like Cells
}

\author{
Yumei Li, ${ }^{1, *}$ Laibing Liu, ${ }^{2, *}$ Zijiang Yu, ${ }^{1}$ Yan Yu, ${ }^{1}$ Baofei Sun, ${ }^{1}$ Chaolun Xiao, ${ }^{1}$ Shipeng Luo, and Lin Li ${ }^{1}$
}

\begin{abstract}
Edaravone can induce differentiation of bone marrow mesenchymal stem cells (BMSCs) into neuron-like cells and replace lost cells by transplanting neuron-like cells to repair spinal cord injury (SCI). In this study, BMSCs were derived from the bone marrow of male Wistar rats $(4$ weeks old) through density gradient centrifugation $(1.073 \mathrm{~g} /$ $\mathrm{mL}$ ), and the cell purity of BMSCs was up to $95 \%$. The combined injection of basic fibroblast growth factor and edaravone was conducted to differentiate BMSCs into neuron-like cells. In this study, 120 male Wistar rats were used to establish the model of semitransverse SCI; on the seventh day, neuron-like cells were labeled by BrdU and then injected into the epicenter of the injury of rats. On the 14th day after cell transplantation, the biotin dextran amine (BDA) fluorescent agent was used to track the repair of nerve damage. At 7, 14, 21, and 30 days after SCI, the Basso, Beattie, and Bresnahan (BBB) locomotor scale method was used to measure the functional recovery of hind limbs in rats. Additionally, hematoxylin and eosin (H\&E) staining, Nissl staining, immunohistochemistry, transmission electron microscopy (TEM), Western blotting, and Real-time quantitative reverse transcripion PCR (qRT-PCR) were used to observe the regeneration of nerve cells. In the edaravone+BMSC group, behavioral analysis of locomotor function showed that functional recovery was significantly enhanced after transplantation of the cells, BrdU-positive cells could be observed scattered in the injured area and extended to both the head and tail, and the BDA tracer shows that the edaravone+BMSC group emits more fluorescent signals. Additionally, H\&E staining, Nissl staining, and immunohistochemistry revealed that the space of spinal cord tissue was attenuated and the neurons were increased. Western blotting and qRT-PCR showed that the expression levels of neuron-specific enolase (NSE), Nestin, and neurofilament 200 (NF) were increased, while the expression of glial fibrillary acidic protein (GFAP) was decreased. TEM showed that cytoplasmic edema was reduced, mitochondrial vacuoles were attenuated, and nuclear chromatin concentration was declined after transplantation of neuron-like cells. Moreover, with the extension of time of edaravone+BMSC transplantation, the structures of mitochondria and endoplasmic reticulum tended to be normal. In summary, the induced differentiation of BMSC transplantation can significantly promote the functional repair of SCI.
\end{abstract}

Keywords: spinal cord injury, bone marrow mesenchymal stem cells, cell transplantation, edaravone, functional recovery

\section{Introduction}

Q PINAL CORD INJURY (SCI) is mainly caused by traffic accidents, falling buildings, and sports, with an annual incidence of about 500,000 cases worldwide (Fan et al., 2017;
Matyas et al., 2017; Xu and Yang, 2019). It is damage to the spinal cord that causes temporary or permanent changes in its function. Symptoms may include loss of muscle function, sensation, or autonomic function in the parts of the body served by the spinal cord below the level of the injury. At the

\footnotetext{
${ }^{1}$ Department of Anatomy, School of Basic Medical Sciences, Guizhou Medical University, Guiyang, China.

${ }^{2}$ Department of Neurosurgery, Affiliated Baiyun Hospital, Guizhou Medical University, Guiyang, China.

*These authors contributed equally to this work.
} 
early stage of injury, damaged blood vessels and cell membranes are destroyed directly and then the secondary cascade reaction triggers production of vascular dysfunction, ischemia, edema, and oxygen free radicals, while promoting the necrosis and apoptosis of adjacent nerve cells (Matyas et al., 2017).

Stem cell therapy, known as one of the most promising treatments, is the use of stem cells to treat or prevent a disease or condition (Namjoo et al., 2018; Pearse et al., 2018). Existing animal experiments showed that embryonic stem cells, neural stem cells, umbilical cord blood stem cells, olfactory ensheathing cells, and Schwann cells play a substantial role in promoting the repair of SCI, while these cells are limited by a variety of ethical and immune rejection issues (Song et al., 2019). Bone marrow mesenchymal stem cells (BMSCs) possess incomparable advantages over other stem cells (Flouzat-Lachaniette et al., 2016; Hernigon et al., 2015; Long et al., 2018). In animal studies, BMSCs have displayed many advantages in the treatment of SCI (Ramalho et al., 2018; Sabirzhanov et al., 2019; Wang et al., 2019). Therefore, exploration of BMSC transplantation for SCI associates with clinical significance and broad research prospects.

Transplantation of specific cells differentiated by pluripotent stem cells can significantly promote the functional repair of SCI. Edaravone was originally developed as a potent free radical scavenger and has been shown to delay neuronal apoptosis, thereby improving nerve function and the microenvironment after SCI. Edaravone can provide a favorable environment for the treatment of SCI using Schwann cell transplantation (Xu and Yang, 2019), which suggests that edaravone is beneficial to the survival of transplanted stem cells. The present study aimed to explore the capability of edaravone inducing MSCs to differentiate into neuron-like cells in vitro. To explore the effects of induced MSCs on the functional recovery of an injured spinal cord, the induced MSCs were transplanted into rats with SCI so as to provide a reliable basis for application of somatic cells in the treatment of SCI.

\section{Materials and Methods}

\section{Cell culture and directional differentiation}

In this study, BMSCs were derived from the bone marrow of male Wistar rats (4 weeks old) through density gradient centrifugation $(1.073 \mathrm{~g} / \mathrm{mL})$. The cells were cultured in Dulbecco's modified Eagle's medium (DMEM; HyClone, Logan, UT) containing $10 \%$ fetal bovine serum (FBS; Gibco, New York, NY) at $37^{\circ} \mathrm{C}$ with $5 \% \mathrm{CO}_{2}$. BMSCs were separated and purified by Percoll medium density gradient centrifugation and repeated subculture and differential adherence methods; $5 \times 10^{4}$ passage 3 BMSCs were seeded in six-well plates and cultured until reaching 50\%-60\% confluency. The cells were randomly divided into three groups:

(1) Normal control (cultivation in DMEM containing $10 \%$ FBS with the same amount of saline).

(2) Cells+basic fibroblast growth factor (bFGF; Abcam, Cambridge, UK)+edaravone injection (Nanjing, China) (ER group; cultivation in DMEM containing 10\% FBS, and $10 \mu \mathrm{g} / \mathrm{L}$ bFGF was added and allowed to incubate for 24 hours, then cells were washed with phosphate-buffered saline (PBS) and treated with different concentrations $(5,10,15,20$,
25 , and $30 \mathrm{mg} / \mathrm{L}$ ) of edaravone in serum-free DMEM for 24 hours, and the optimal intervention concentration was determined based on cell survival and morphological alterations).

(3) Cells $+\beta$-mercaptoethanol (Abcam)+all-trans retinoic acid (ATRA); (Abcam) (ATRA group; cultivation in DMEM containing $10 \%$ FBS, and $1 \mathrm{mmol} / \mathrm{L} \quad B-$ mercaptoethanol was added and allowed to incubate for 24 hours, then cells were washed with PBS and treated with $1 \mathrm{mmol} / \mathrm{L}$ ATRA in serum-free DMEM for 24 hours).

\section{Experimental animals}

Specific pathogen-free, male Wistar rats (body weight, $180 \pm 20 \mathrm{~g}$ ), obtained from the Army Medical University (Chongqing, China), were maintained at the Animal Center of Guizhou Medical University (Guizhou, China). All animals had free access to food and water and were kept in comfortable/clean cages at $25^{\circ} \mathrm{C}-27^{\circ} \mathrm{C}$. Upon successful establishment of the animal model, animals were executed according to requirements of the Ethics Committee of Guizhou Medical University.

\section{Establishment of animal model}

Briefly, rats were anesthetized with $1 \%$ sodium pentobarbital $(40 \mathrm{mg} / \mathrm{kg}$; Kemiou, Tianjin, China), thoracic medulla segments of T8-T10 were fully exposed by costal arch, the spinal cord in the right half of the posterior median sulcus was transected at $\sim$ T9 with a sharp blade, and as a result, the rat model of SCI was established successfully. On the fourth day of SCI, P3 generation cells were added with $20 \mu \mathrm{mol} / \mathrm{L} \mathrm{BrdU}(20 \mu \mathrm{L})$-labeled BMSCs to track the survival rate of cells after transplantation. On the seventh day of SCI, the experimental animals were randomly divided into three groups:

(1) The normal control group $(n=40$, slowly inject $0.9 \%$ saline solution into the spinal cord parenchyma $1 \mathrm{~mm}$ from the head and tail of the SCI area and $0.5 \mathrm{~mm}$ from the midline, with a total of four injections on both sides; the injection depth is $1.25 \mathrm{~mm}, 3 \mu \mathrm{L}$ per point).

(2) The BMSC transplantation group ( $n=40$, slowly inject the BMSC suspension induced by DMEM into the spinal cord parenchyma $1 \mathrm{~mm}$ from the head and tail of the SCI area and $0.5 \mathrm{~mm}$ from the midline, with a total of four injections on both sides; the injection depth is $1.25 \mathrm{~mm}, 3 \mu \mathrm{L}$ per point [100,000 cells]).

(3) The edaravone+BMSC transplantation group $(n=40$, slowly inject the BMSC suspension induced by edaravone injection into the spinal cord parenchyma $1 \mathrm{~mm}$ from the head and tail of the SCI area and $0.5 \mathrm{~mm}$ from the midline, with a total of four injections on both sides; the injection depth is $1.25 \mathrm{~mm}, 3 \mu \mathrm{L}$ per point [100,000 cells]).

After transplantation, animals were injected with cyclosporin A $[2.5 \mathrm{mg} /(\mathrm{kg} \cdot 12$ hours $)$, continued for 48 hours, Shanghai, China] into the abdominal cavity to counteract the immune response, and 4 periods of $7,14,21$, and 30 days were continuously monitored. On the 14th day after cell transplantation, the biotin dextran amine (BDA) fluorescent agent was used to track the repair of nerve damage, and gentamicin $[0.8 \mathrm{mg} /(100 \mathrm{~g} \cdot$ day $)]$ was injected intraperitoneally for 7 days after the operation to prevent infection. Subsequently, rats were killed according to the ethical regulations. 


\section{Histopathological and immunohistochemical analyses}

Briefly, formalin-fixed, paraffin-embedded spinal cord tissues of rats were sliced into 5- $\mu \mathrm{m}$ sections and subjected to hematoxylin and eosin (H\&E; Solebao, Beijing, China) and Nissl staining (Solebao) for histological assessment. In addition, BMSCs and tissue sections were fixed with $4 \%$ paraformaldehyde after corresponding treatment and incubated with anti-CD34/44/45 (Abcam) and NSE/GFAP/ Nestin/NF/BrdU (Santa Cruz Biotechnology, Inc., Dallas, TX). The images were acquired by two pathologists who were blinded to the treatment using a Leica digital microscope (Leica Camera AG, Wetzlar, Germany).

\section{Flow cytometry}

The primary cells were centrifuged at $1500 \mathrm{rpm}$ for 10 minutes. Cell suspensions were prepared with concentration of $1 \times 10^{7}$ cells/mL. CD90, CD29, CD44, CD45, and CD34 antibodies were used at a dilution ratio of $1: 100$, respectively. The resulting cells were filtered through a 70-mm cell strainer (BD Biosciences, San Jose, CA) and analyzed with FACSCanto II (BD Biosciences).

\section{Scanning electron microscopy}

BMSCs were incubated for 24 hours, and the cells were cultured until reaching confluency of $90 \%$. Then, cells were dehydrated with an alcohol gradient, and morphological changes of cells were observed under a scanning electron microscope (Hitachi, Tokyo, Japan).

\section{Western blot analysis}

The protein concentration was estimated using a bicinchoninic acid (BCA) protein assay kit (Beyotime Institute of Biotechnology, Shanghai, China). Protein samples were resolved by sodium dodecyl sulfate-polyacrylamide gel electrophoresis (SDS-PAGE) and transferred onto polyvinylidene difluoride (PVDF) membranes (Millipore, Burlington, MA). Then, the membranes were blocked with $5 \%$ skim milk and probed overnight at $4{ }^{\circ} \mathrm{C}$ with antibodies targeting NSE/GFAP/Nestin/NF/BrdU (dilution, 1:500; Santa Cruz Biotechnology, Inc.) and $\beta$-actin (dilution, 1:6000; Abcam). Subsequently, membranes were incubated with the corresponding secondary antibody (Abcam) at room temperature for 1 hour. Immunoreactive bands were detected using an enhanced chemiluminescence system (Clarity Western ECL Substrate; Bio-Rad Laboratories, Inc., Hercules, CA) and quantified on a gel imaging system (Bio-Rad Laboratories, Inc.).

\section{RNA extraction and Real-time quantitative reverse transcripion PCR (qRT-PCR)}

Total RNA was extracted from the lungs and cells using the TRIzol reagent (Invitrogen) and reverse transcribed using oligo-dT primers and reverse transcriptase (Tiangen, Beijing, China). qRT-polymerase chain reaction was performed using the SYBR Green qPCR Mix Kit (Tiangen) and the corresponding primers (Table 1).

\section{BDA tracer}

On the 14th day after cell transplantation, after the animals were anesthetized, the median incision was made to expose the skull, and the center of the anterior fontanelle was used as a marker point. Ten holes of $\sim 0.5 \mathrm{~mm}$ depth from the surface of the anterior fontanelle were drilled with a dental electric drill at $1.0 \mathrm{~mm}$ before/after and $1.5 \mathrm{~mm}$ from the midline to slowly inject $0.2 \mu \mathrm{L}$ of $10 \%$ BDA solution into the cerebral cortex. First, the subcutaneous injection depth was at $2.0 \mathrm{~mm}$, then the needle was slowly withdrawn to $1.0 \mathrm{~mm}$ subcutaneous depth to continue the injection; the total amount of BDA was $4 \mu \mathrm{L}$.

\section{The Basso, Beattie, and Bresnahan locomotor scale method}

After transplantation, evaluation of behavioral function of experimental animals was performed after 7, 14, 21, and 30 days. The Basso, Beattie, and Bresnahan (BBB) locomotor scale method was used for behavioral evaluation of rats. The observers were nonexperimenters who were familiar with scoring criteria and blinded to the experiments. The observation period of the $\mathrm{BBB}$ score was 4 minutes.

\section{Transmission electron microscopy}

Spinal cord tissues (1-mm segments) were excised, fixed with $2 \%$ glutaraldehyde for 2 hours, dehydrated with ethanol, embedded into paraffin, sliced by an LKBV ultrathin slicer (Leica, Germany), stained, and observed under a transmission electron microscope (Hitachi).

\section{Statistical analysis}

All experimental data are expressed as mean \pm standard error of the mean (SEM) and plotted using GraphPad Prism 5.0 software (GraphPad Software, Inc., San Diego, CA). All experimental data were statistically analyzed using SPSS 17.0 software (IBM, Armonk, NY). $p$-Value $<0.05$ was considered statistically significant.

TABLE 1.

\begin{tabular}{llc}
\hline Gene & \multicolumn{2}{c}{ The sequences of the primer $\left(5^{\prime}-3^{\prime}\right)$} \\
\hline NSE & Forward:GGCACTCTACCAGGACTTIG & Reverse:GCGATGACTCACCATAACCC \\
GFAP & Forward:TGGCCACCAGTAACATGCAA & Reverse:CAGTTGGCGGCGATAGTCAT \\
Nestin & Forward:AGCTGGCGCACCTCAAGATG & Reverse:AGGGAAGTTGGGCTCAGGAC \\
NF & Forward:CAGAGCTGGAGGCACTGAA & Reverse:CATCTCCCACTTGGTGTTCC \\
$\beta$-actin & Forward:CATGGGTCAGAAGGATTCCT & Reverse:TGATCTGGGTCATCTTCTCG \\
\hline
\end{tabular}




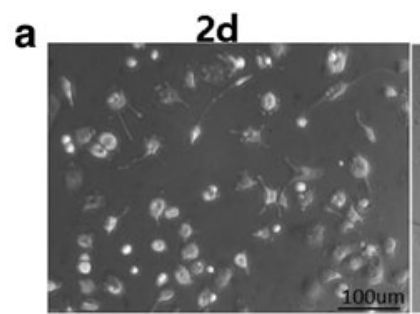

$4 d$

9d

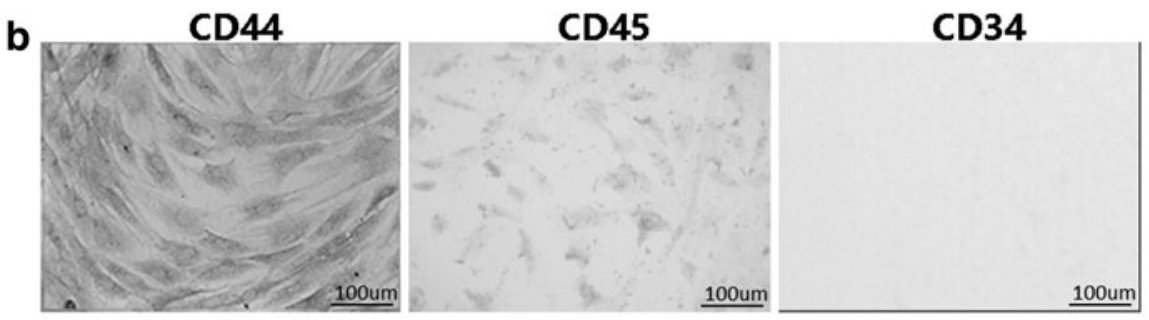

C
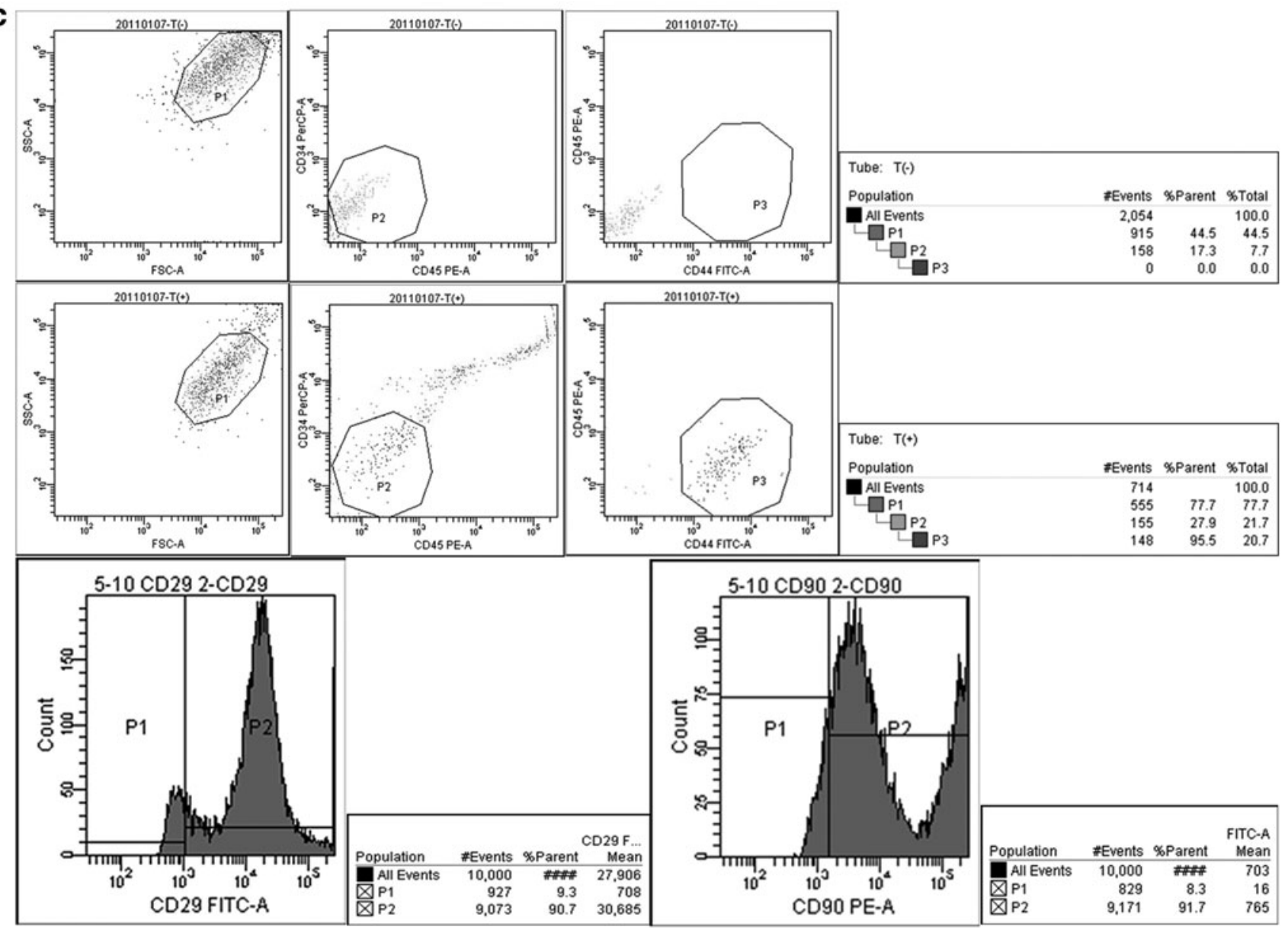

FIG. 1. Detection of primary cultured cells: (a) Morphological changes of cells at different periods of cultivation (magnification, $\times 200$ ). (b) Immunohistochemistry of CD34, CD44, and CD45 in BMSCs (magnification, $\times 200$ ). (c) Testing the expression of specific markers in BMSCs by flow cytometry. BMSCs, bone marrow mesenchymal stem cells.

\section{Results}

\section{Purity and phenotype of primary BMSCs}

The cultured primary BMSCs were rounded and similar to fibroblasts, which could be cultured for 7-9 days, with a single uniform morphology and fascicular arrangement (Fig. 1a). Using the third generation of BMSCs, CD44 was positively expressed in the majority of cells, while CD34 and CD45 were not expressed based on immunohistochemical staining (Fig. 1b). Results of flow cytometry showed that the CD44-positive rate of BMSCs was $95.5 \%$, the positive rate for CD90 was $91.7 \%$, and the positive rate for CD29 was $90.7 \%$, indicating that characteristic surface antigens of mesenchymal stem cells (MSCs) were expressed, whereas 

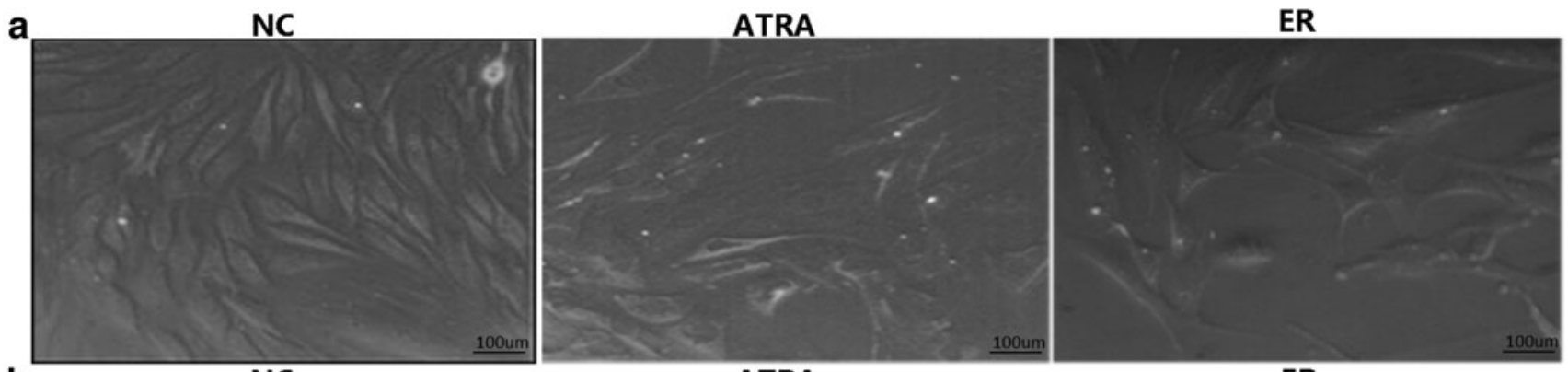

b

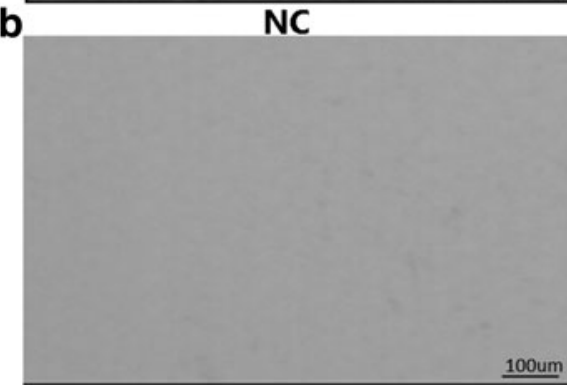

ATRA
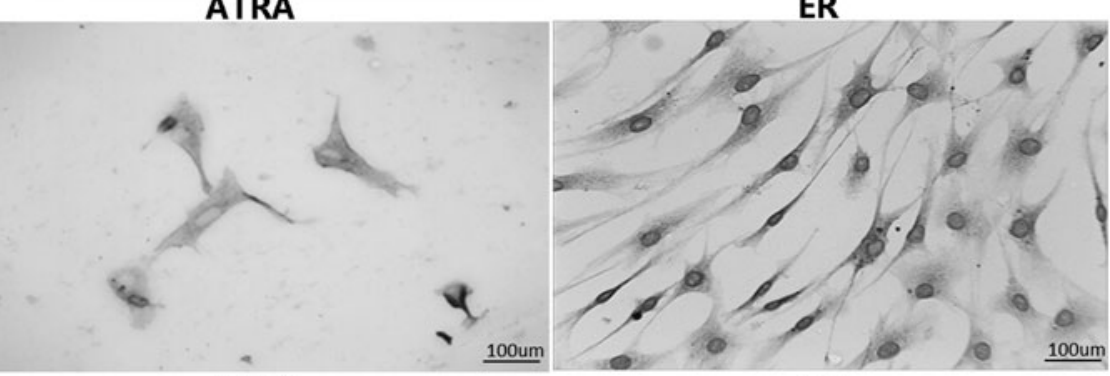

C

NC

ATRA

ER
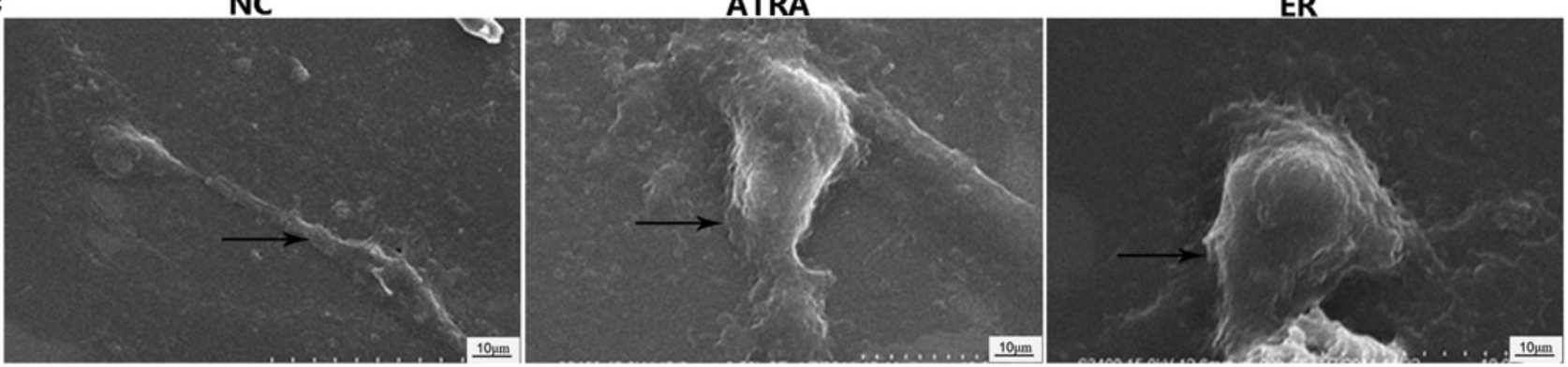

FIG. 2. Detection of BMSCs after induced differentiation into neuron-like cells: (a) Morphological changes of induced BMSCs (magnification, $\times 200$ ). (b) Immunohistochemistry of NSE in induced BMSCs (magnification, $\times 400)$. (c) The morphology of BMSCs treated with edaravone was observed by SEM (bar $=10 \mu \mathrm{m})$. Arrows indicate changes of cells body after induction. NSE, neuron-specific enolase; SEM, scanning electron microscope.

CD34 and CD45 were not expressed (Fig. 1c). These results confirmed that high-purity BMSCs were obtained.

\section{Edaravone injection induced differentiation of BMSCs into neuron-like cells}

After 24 hours of preinduction with bFGF, some cells became smaller and rounded, and when edaravone was injected, the cells began to retract into a polygonal shape, and the presence of large protuberant structures was noted on a number of cells. After induction for 5 hours, the bipolar, multipolar, and conical shapes of neuron-like cells were observed, and the number of typical neuron-like cells no longer increased until induction for 6 hours (Fig. 2a), and the cells could adhere to the wall after 24 hours of edaravone injection (data not shown). The morphological features of cells were similar to the edaravone group, while numerous cells died in the positive control (ATRA) group, and no change in the normal control group was found (Fig. 2a).

The induction rates of BMSCs at different liquid concentrations were measured, and the morphological features of BMSCs did not change remarkably when the concentration was 5 or $10 \mathrm{mg} / \mathrm{L}$. When cells differentiated into neural-like cells at a concentration of $20 \mathrm{mg} / \mathrm{L}$ and higher, cells died due to rapid differentiation (data not shown), and the optimal concentration of induction was found to be $20 \mathrm{mg} / \mathrm{L}$ (Fig. 2a).

FIG. 3. Induction of neuron-like cells promoted the recovery of motor function after SCI in rats. (a) Histological changes in the injured spinal cord tissue in each group were assessed by hematoxylin and eosin and Nissl staining methods (magnification, $\times 400$ ). (b) Immunohistochemistry for NSE, Nestin, GFAP, NF, and BrdU in the spinal cord tissue in different groups (magnification, $\times 400$ ). Data are presented as mean \pm standard deviation (SD), $n=6, * p<0.05$ versus the NC group, ${ }^{\#} p<0.05$ BMCs-21d, and ${ }^{\&} p<0.05$ BMCs-30d. NC: normal control group; BMCs-21d: BMSCs were transplanted for 21 days; BMCs-30d: BMSCs were transplanted for 30 days; ER+BMCs-14d: BMSCs were induced by edaravone and then transplanted for 14 days; ER+BMCs-21d: BMSCs were induced by edaravone and then transplanted for 21 days; ER+BMCs-30d: BMSCs were induced by edaravone and then transplanted for 30 days. GFAP, glial fibrillary acidic protein; NF, neurofilament 200; SCI, spinal cord injury. 
a

NC

BMCs-21d BMCs-30d ER+BMCs-14d ER+BMCs-21d ER+BMCs-30d

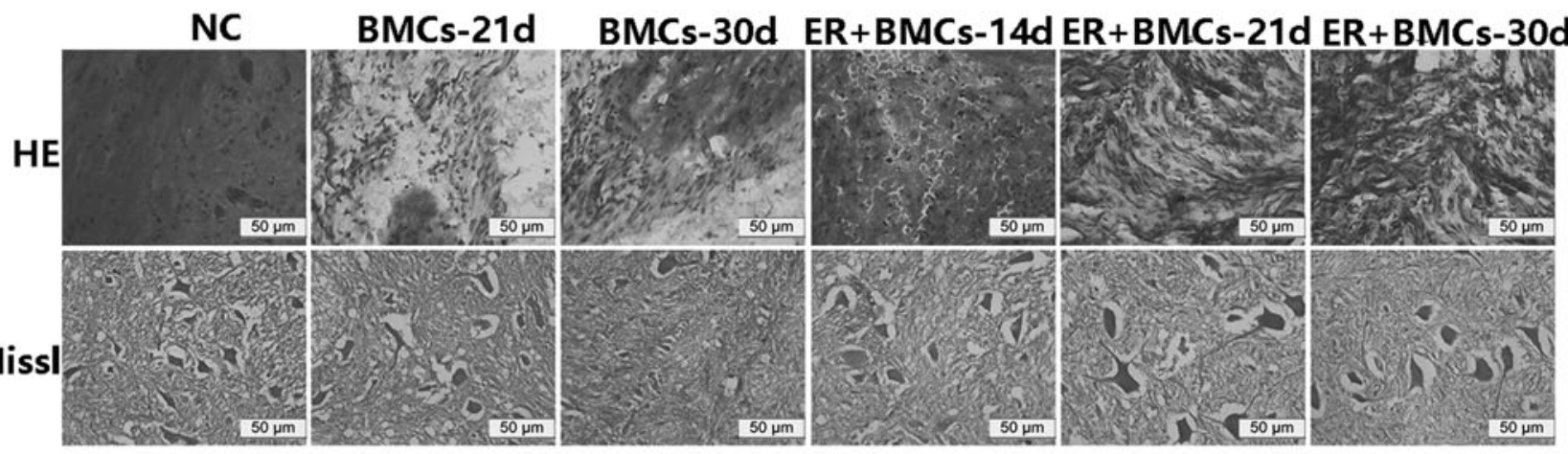

b

NC BMCs-21d

BMCs-30d ER+BMCs-14d ER+BMCs-21d ER+BMCs-30d

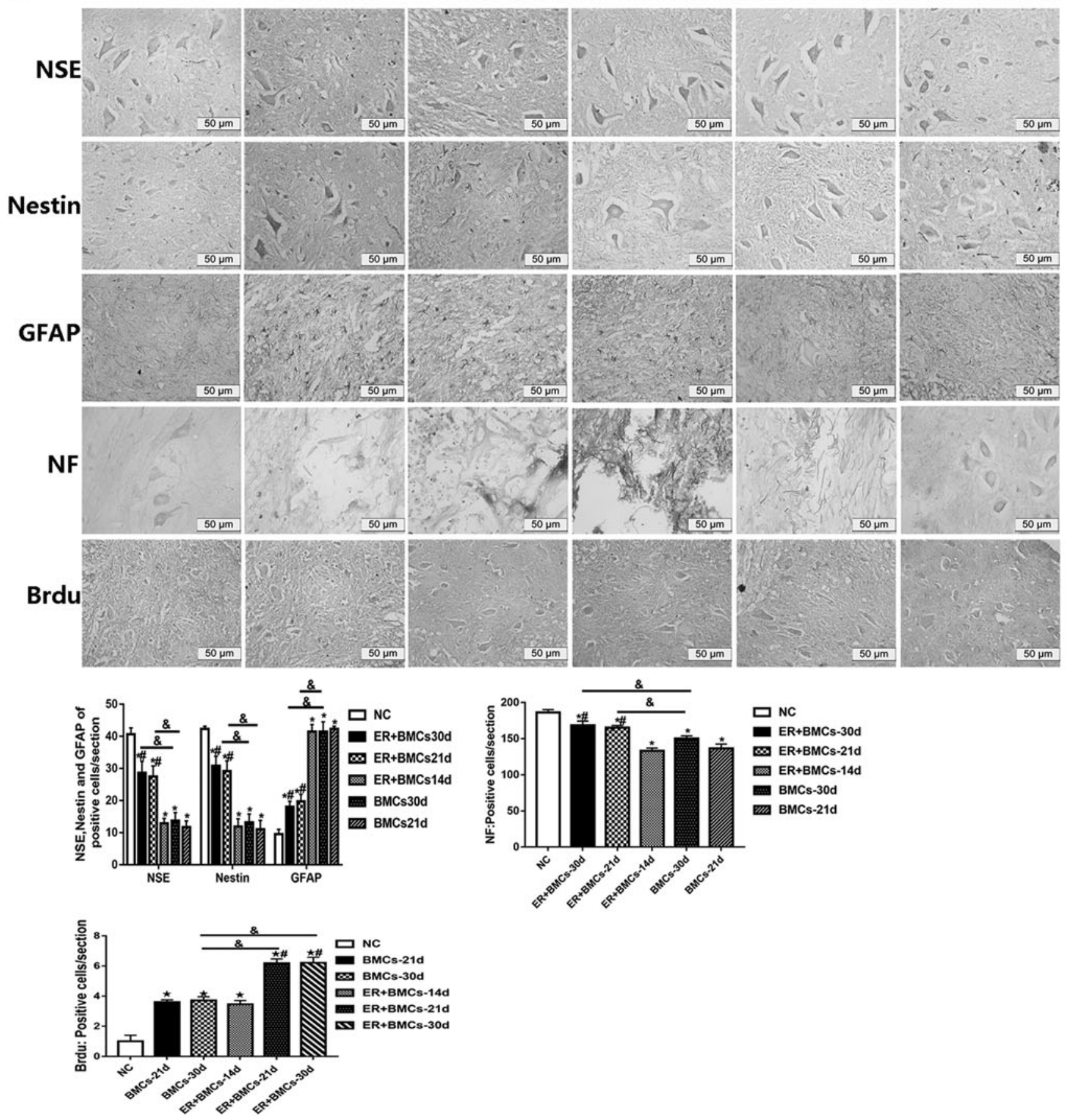


After induction, neuron-specific enolase (NSE) was positively expressed in the edaravone group, where the positive rate of NSE was about $98.6 \%$, and its expression level was lower in the positive control (ATRA) group, where the positive rate of NSE was only about $68 \%$, while it was not expressed in the normal control group (Fig. 2b). SEM showed that the cells shrank around the nucleus and the body extended an axon-like structure in the edaravone group. Besides, morphological changes were lower in the positive control group, while those changes were not observed in the normal control group, and the MSCs had typical spindle-shaped morphology. It was revealed that edaravone could effectively induce differentiation into nervelike cells (Fig. 2c).

\section{Transplantation of induced BMSCs noticeably promoted functional repair of injured hind limbs}

In the BMSC group, the structures of gray matter and white matter were changed, and a limited number of neurons were regenerated in the inflammatory cavity 21 days after the injury. It also was revealed that the repair was not apparent after 30 days; however, the structures of gray matter and white matter were arranged in a regular manner 14 days after injury in the edaravone+BMSC transplantation group. Additionally, necrosis in the cavity gradually shrank due to regeneration of neurons, and gray matter and white matter are more orderly with the extension of transplantation time in the edaravone+BMSC transplantation group 21 days after injury as shown by H\&E and Nissl staining methods, whereas the repair was superior after 30 days (Fig. 3a).

The expression levels of NSE, neurofilament 200 (NF), Nestin, and glial fibrillary acidic protein (GFAP) were measured to assess the degree of recovery after SCI by immunohistochemical analysis. It was noted that the expression levels of NSE, Nestin, and NF were increased; the positive rate of NSE was about $68 \%$, which was much higher than $38 \%$ of the BMSC group; and the positive rates of Nestin and NF were $78 \%$ and $95 \%$, respectively, which were stronger than $37 \%$ and $82 \%$ of the BMSC group. The GFAP was highly expressed in the BMSC group, the positive rate of GFAP was about 59\%; however, the rate was about $28 \%$ in the edaravone+BMSC group (Fig. 3b).

The change of expression level was more remarkable with the extension of time of edaravone+BMSC transplantation, indicating that the number of regenerated neurons gradually increased, whereas the number of glial cells gradually decreased; besides, the arrangement of nerve fibers was gradually standardized, the space between spinal cord tissues gradually decreased due to the filling of regenerated neurons, and the motor function gradually improved in the edaravone+BMSC transplantation group 21 and 30 days after transplantation (Fig. 3b) $(p<0.05)$. In the spinal cord of transplanted rats, BrdU-positive cells could be observed scattered in the injured area and extended to both the head and tail, and the edaravone+BMSC group showed the most cells. The BDA tracer showed that the edaravone+BMSC group emits more fluorescent signals and repairs nerve damage (Fig. 4a).

Results of Western blotting and qRT-PCR showed that the expression level of GFAP was significantly higher in the
BMSC group, and the expression levels of NSE, Nestin, and $\mathrm{NF}$ were markedly higher in the edaravone+BMSC group compared with other groups $(p<0.05)$ (Fig. 4c, d). Results of transmission electron microscopy revealed that the neuronal nuclear membrane was intact, heterochromatin was concentrated and gathered around the nuclear membrane, cytoplasm was edematous, endoplasmic reticulum was swollen, mitochondrial cristae were fractured, and some of them showed vacuolar changes in the BMSC group.

However, after transplantation with edaravone+BMSCs, the nuclear heterochromatin of nerve cells was reduced and cytoplasmic edema and changes in mitochondrial vacuoles were decreased. Meanwhile, the nucleus of nerve cells was oval, and the structure of mitochondria and endoplasmic reticulum tended to be normal with the extension of transplantation time (Fig. 4e).

\section{BBB score}

After spinal cord semitransverse injury, the kinetic energy of the right hind limb of rats was lost in a short period of time, and the BBB score was 0. After 1-3 days of injury, rats did not recover. Before transplantation, the $\mathrm{BBB}$ score in each group reached about 4 . The majority of rats began to develop contracture in the injured hind limb, and movement of the contralateral hind limb was slightly limited. With the extension of the time of transplantation, the injured hind limb was repaired slowly in the BMSC transplantation group. However, the functional recovery of injured hind limbs was significantly faster and the repair effect was noticeably better in the edaravone+BMSC transplantation group (Fig. 4b).

\section{Discussion}

The loss of neurons and glial cells can be considered as one of the main causes of permanent functional dysfunction after SCI, in which various cell transplants need to be used to compensate the loss of neurons and glial cells, guide axonal growth, and treat the SCI (Sabirzhanov et al., 2019; Li et al., 2017). In the present study, high-purity BMSCs obtained in vitro were transplanted into the injured spinal cord after induction of neuron-like cells by edaravone. We found that the transplanted BMSCs could promote the recovery of motor function and injured spinal cord and play a protective role in the nerves.

BMSCs possess the advantages of a stable genetic background, no immune rejection, and no ethical disputes after transplantation. BMSCs can be isolated and cultured to large numbers from a small volume of bone marrow and are therefore sources of cells for bone tissue engineering applications and they have become the main source of stem cells for transplantation in disorders of the nervous system (Abdallah et al., 2019; De Becker and Riet, 2016; Kurose et al., 2019; Lu et al., 2019; Takahashi et al., 2018; Yousefifard et al., 2019). In the present research, CD44, CD34, CD29, CD90, and CD45 were selected to test cultured BMSCs. The results showed that the cultured third-generation cells expressed cell surface antigens, CD44, CD29, and CD90, stably, while that expression was not observed in CD34 and CD45, demonstrating that the obtained cells were nonhematopoietic stem cells with high purity. 

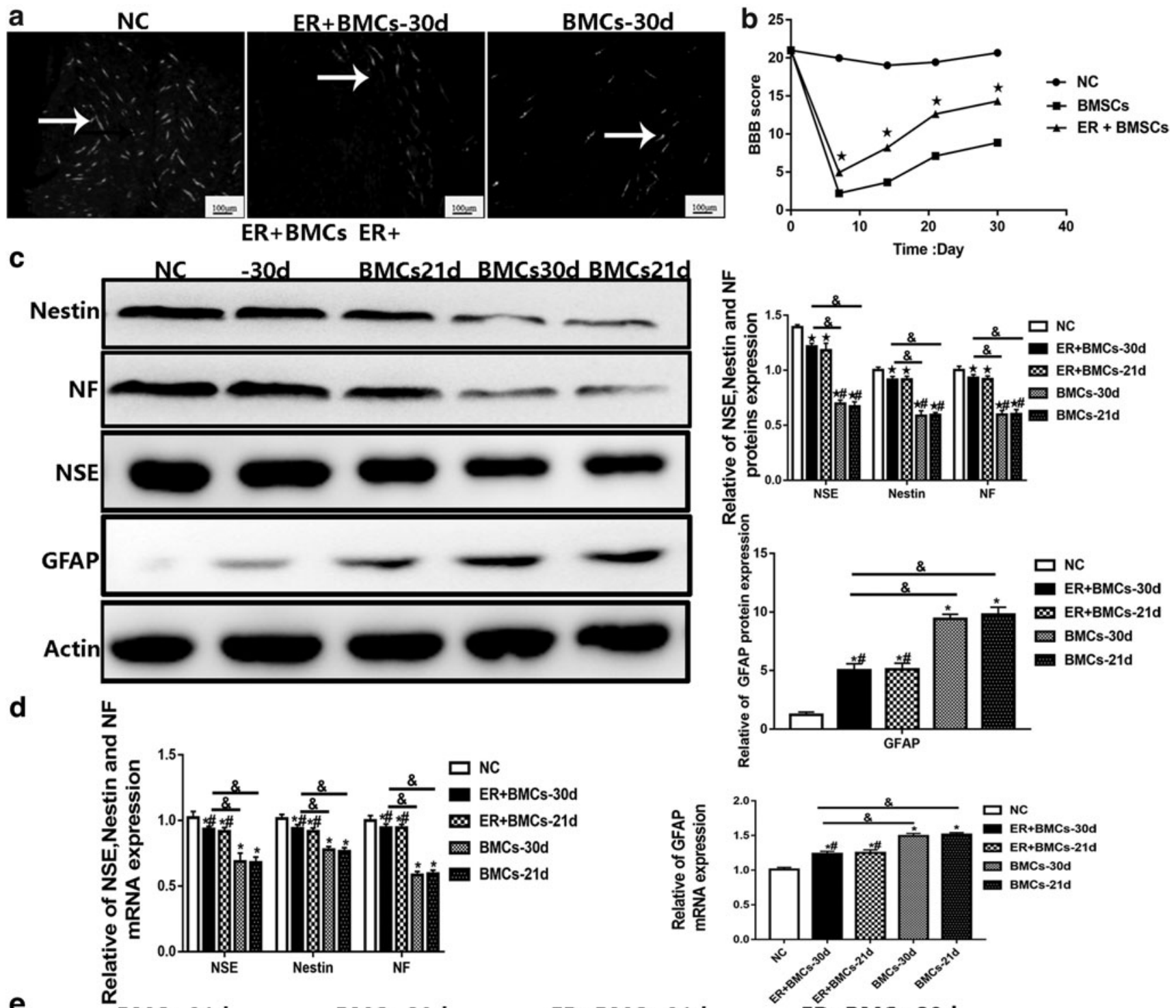

e

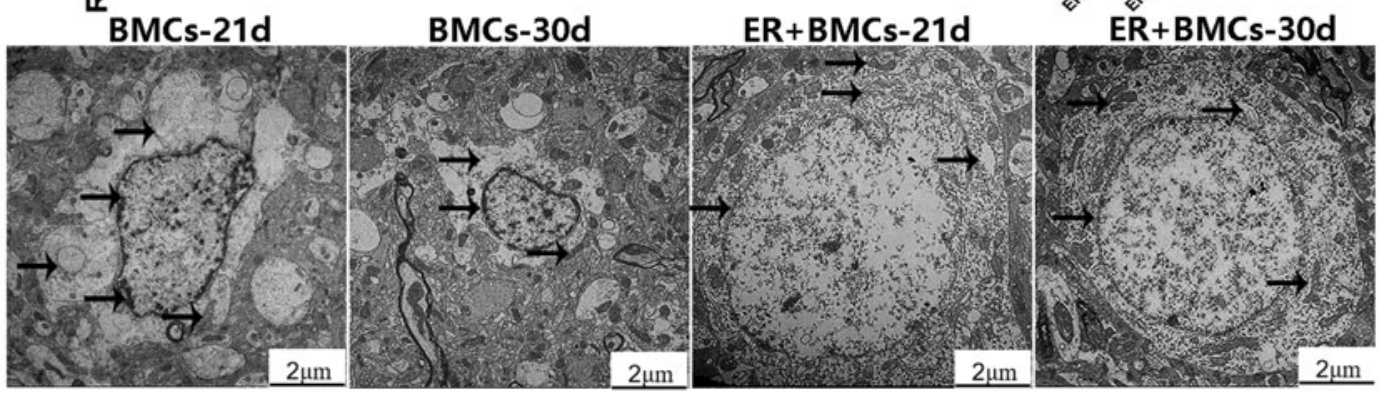

FIG. 4. Induction of neuron-like cells promoted the recovery of motor function after SCI in rats. (a) The BDA fluorescent agent was used to track the repair of nerve damage. (b) Results of the BBB score for motor function of experimental animals with transplantation of DMEM and BMSCs. NC: normal control group; BMCs: BMSCs were transplanted; and ER+BMCs: BMSCs were induced by edaravone and then transplanted. (c) Protein levels of NSE, Nestin, NF, and GFAP in spinal cord tissue in each group were assessed by immunoblot. Quantitation was performed using Image Pro Plus 6.0 and QuantityOne software. Data are presented as mean $\pm \mathrm{SD}, n=6, * p<0.05$ versus the NC group, ${ }^{*} p<0.05 \mathrm{BMCs}-21 \mathrm{~d}$, and ${ }^{*} p<0.05$ BMCs-30d. (d) Expression level of NSE, Nestin, NF, and GFAP genes in spinal cord tissue in each group as assessed by qRT-PCR. Data are presented as mean $\pm \mathrm{SD}, n=6, * p<0.05$ versus the NC group, ${ }^{*} p<0.05$ BMCs-21d, and ${ }^{\&} p<0.05$ BMCs-30d. (e) Structural changes of nerve cells in spinal cord tissue were observed by TEM $(b a r=2 \mu \mathrm{m})$. NC: normal control group, BMCs-21d: BMSCs were transplanted for 21 days; BMCs-30d: BMSCs were transplanted for 30 days; ER+BMCs-21d: BMSCs were induced by edaravone and then transplanted for 21 days; ER+BMCs-30d: BMSCs were induced by edaravone and then transplanted for 30 days. Arrows indicate changes in the nuclear membrane, endoplasmic reticulum and mitochondria of neurons. BBB, Basso, Beattie, and Bresnahan; BDA, biotin dextran amine; DMEM, Dulbecco's modified Eagle's medium; qRT-PCR, Real-time quantitative reverse transcription PCR; TEM, transmission electron microscopy. 
BMSCs can not only differentiate into fat cells, chondroblasts, osteoblasts, and myoblasts but also differentiate into nerve cells, glial cells, hepatocytes, and cardiomyocytes, etc., and they have the potential of multidirectional differentiation under an appropriate microenvironment (Lin et al., 2018; Zhang et al., 2018). In the current study, bFGF combined with edaravone was used to induce BMSCs for differentiating into neuron-like cells. It is also noteworthy that bFGF is known to stimulate angiogenesis and thus to influence proliferation, migration, and survival of tumor cells and it can promote self-proliferation and differentiation of neural stem cells (Maric et al., 2007).

Edaravone is a hydroxyl free radical scavenger and antioxidant, which can inhibit lipid peroxidation, delay neuron death, and prevent vascular endothelial cell injury, as well as play an anti-ischemic role (Xu and Yang, 2019). Normally, glial cells need to survive in a medium containing a certain amount of serum, while that is not essential for cultivation of neurons. Therefore, we hypothesized that serum-free induced fluid could promote differentiation of BMSCs into neurons rather than glial cells. The results achieved in the present study showed that BMSCs after induction had typical neuron-like cell morphology. Besides, increased expression of NSE, NF, and Nestin and decreased expression of GFAP were noted, suggesting that the combination of bFGF and edaravone can promote induced differentiation of BMSCs into neuron-like cells rather than glial cells.

In addition, there are two key characteristics of BMSCs that make them ideal for clinical use in improvement of function following SCI. One is that BMSCs protect the injured CNS from further cellular damage. The other is that BMSCs support nerve fiber regeneration (Han et al., 2015; Vilaça-Faria et al., 2019). The current research revealed that compared with the BMSC group, the number of neurons in the damaged spinal cord tissues in the edaravone+BMSC group was gradually increased, while the number of glial cells gradually decreased, and the space in the spinal cord tissues gradually decreased due to the filling of proliferated nerve tissues. The results of the experiments were consistent with our expectations.

The mechanism of improving the function of BMSCs in the treatment of SCI can be explained by the following: first, the successful induction of neuron-like cell transplantation penetrates and fuses into the lesion tissue, replaces damaged cells, rebuilds the nerve pathway, and achieves the purpose of restoring nerve function; and second, studies have confirmed that edaravone can provide a favorable environment for the treatment of SCI using Schwann cell transplantation (Xu and Yang, 2019), such as secretion of the nerve growth factor, brain-derived nerve growth factor, and other neurotrophic factors and neurotransmitters (Badner et al., 2017; Dalamagkas et al., 2018; Xiao et al., 2018), which may also be the reason that transplantation after induction with edaravone can promote the repair of the injured spinal cord.

BMSCs can produce some cytokines, which may play a positive role in the recovery of the injured spinal cord in the BMSC group, but the effect is not particularly obvious. However, the types of growth factors that edaravone can secrete and the specific mechanism of repair still need to be further studied.
Taken together, the induced differentiation of BMSC transplantation can significantly promote functional repair and these are promising cells for treating SCI.

\section{Ethics Approval}

All procedures for the treatment of animals were approved by the Ethics Committee of Guizhou Medical University.

\section{Availability of Data and Materials}

The datasets used and/or analyzed during the current study are available from the corresponding author on reasonable request.

\section{Author Disclosure Statement}

The authors declare they have no competing financial interests.

\section{Funding Information}

The present study was financially supported by the National Natural Science Foundation of China (Grant Nos. 30660189 and 81660002), in addition to the Science and Technology Special Commissioner System Project in Guizhou Province (Grant No. SY [2009] 3058).

\section{References}

Abdallah, B.M., Alzahrani, A.M., Abdel-Moneim, A.M., Ditzel, N., and Kassem, M. (2019). A simple and reliable protocol for long-term culture of murine bone marrow stromal (mesenchymal) stem cells that retained their in vitro and in vivo stemness in long-term culture. Biol. Proced. 21, 3.

Badner, A., Siddiqui, A.M., and Fehlings, M.G. (2017). Spinal cord injuries: How could cell therapy help. Expert Opin. Biol. Ther. 17, 529-541.

Dalamagkas, K., Tsintou, M., and Seifalian, A.M. (2018). Stem cells for spinal cord injuries bearing translational potential. Neural. Regen. Res. 13, 35-42.

De Becker, A., and Riet, I.V. (2016). Homing and migration of mesenchymal stromal cells: How to improve the efficacy of cell therapy? World J. Stem Cells 8, 73-87.

Fan, X., Wang, J.Z., Lin, X.M., and Zhang, L. (2017). Stem cell transplantation for spinal cord injury: A meta-analysis of treatment effectiveness and safety. Neural. Regen. Res. 12, 815-825.

Flouzat-Lachaniette, C.H., Heyberger, C., Bouthors, C., Roubineau, F., Chevallier, N., Rouard, H., and Hernigou, P. (2016). Osteogenic progenitors in bone marrow aspirates have clinical potential for tibial non-unions healing in diabetic patients. Int. Orthop. 40, 1375-1384.

Han, D., Wu, C., Xiong, Q., Zhou, L., and Tian, Y. (2015). Anti-inflammatory mechanism of bone marrow mesenchymal stem cell transplantation in rat model of spinal cord injury. Cell Biochem. Biophys. 71, 1341-1347.

Hernigon, P., Guisson, I., and Homma, Y. (2015). Percutaneous injection of bone marrow mesenchymal stem cells for ankle non-unions decreases complications in patients with diabetes. IntOrthop. 10, 32-39.

Kurose, T., Takahashi, S., Otsuka, T., Nakagawa, K., Imura, T., Sueda, T., and Yuge, L. (2019). Simulated microgravity- 
cultured mesenchymal stem cells improve recovery following spinal cord ischemia in rats. Stem Cell Res. 41, 101601.

Li, X.M., Meng, J., Li, L.T., Guo, T., Yang, L.K., Shi, Q.X., Li, X.B., Chen, Y., Yang, Q., and Zhao, J.N. (2017). Effect of ZBD-2 on chronic pain,depressive-like behaviors and recovery of motor function following spinal cord injury in mice. Behav. Brain Res. 322, 92-99.

Lin, L.,Lin, H., Bai, S., Zheng, L., and Zhang, X. (2018). Bone marrow mesenchymal stem cells (BMSCs) improved functional recovery of spinal cord injury partly by promoting axonal regeneration. Neurochem. Int. 115, 80-84.

Long, M., Cai, L., Li, W., Zhang, L., Guo, S., Zhang, R., Zheng, Y., Liu, X., Wang, M., Zhou, X., Wang, H., Li, X., Li, L., Zhu, Z., Yang, G., and Zheng, H. (2018). DPP-4 inhibitors ImproveDiabetic wound healing via direct and indirect promotion of epithelial-mesenchymal transition and reduction of scarring. Diabetes. 67, 518-531.

Lu, Y., Xing, J., Yin, X., Zhu, X., Yang, A., Luo, J., Gou, J., Dong, S., Xu, J., and Hou, T. (2019). Bone marrow-derived $\mathrm{CD} 44^{+}$cells migrate to tissue-engineered constructs via SDF1/CXCR4-JNK pathway and aid bone repair. Stem Cells Int. 1513526, DOI:10.1155/2019/1513526.

Maric, D., Fiorio Pla, A., Chang, Y.H., and Barker, J.L. (2007). Self-renewing and differentiatingproperties of cortical neural stem cells are selectively regulated by basic fibroblast growth factor (FGF) signaling via specific FGF receptors. J. Neurosci. 27, 1836-1852.

Matyas, J.J., Stewart, A.N., Goldsmith, A., Nan, Z.H., Skeel, R.L., Rossignol, J., and Dunbar, G.L. (2017). Effects of bone-marrowderived MSC transplantation on functional recovery in a rat model of spinal cord injury: Comparisons of transplant locations and cell concentrations. Cell Transplant. 26, 1472-1482.

Namjoo, Z., Moradi, F., Aryanpour, R., Piryaei, A., Joghataei, M.T., Abbasi, Y., Hosseini, A., Hassanzadeh, S., Taklimie, F.R., Beyer, C., and Zendedel, A. (2018). Combined effects of rat Schwann cells and $17 \beta$-estradiol in a spinal cord injury model. Metab. Brain Dis. 33, 1229-1242.

Pearse, D.D., Bastidas, J., Izabel, S.S., and Ghosh, M. (2018). Schwann cell transplantation subdues the pro-inflammatory innate immune cell after spinal cord injury. Int. J. Mol. Sci. 19, E2550.

Ramalho, B.D.S., Almeida, F.M., Sales, C.M., de Lima, S., and Martinez, A.M.B. (2018). Injection of bone marrow mesenchymal stem cells by intravenous or intraperitoneal routes is a viable alternative to spinal cord injury treatment in mice. Neural. Regen. Res. 13, 1046-1053.

Sabirzhanov, B., Matyas, J., Coll-Miro, M., Yu, L.L., Faden, A.I., Stoica, B.A., and Wu, J. (2019). Inhibition of microRNA-711 limits angiopoietin-1 and Akt changes,tissue damage,and motordysfunction after contusive spinal cord injury in mice. Cell Death Dis. 10, 839.
Song, X., Dai, J., Li, H., Li, Y., Hao, W., Zhang, Y., Su, L., and Wei, H. (2019). Anti-aging effects exerted by Tetramethylpyrazine enhances self-renewal and neuronal differentiation of rat bMSCs by suppressing NF-kB signaling. Biosci. Rep. 39, BSR20190761.

Takahashi, S., Nakagawa, K., Tomiyasu, M., Nakashima, A., Katayama, K., Imura, T., Herlambang, B., Okubo, T., Arihiro, K., Kawahara, Y., Yuge, L., and Sueda, T. (2018). Mesenchymal stem cell-based therapy improves lower limb movement after spinal cord ischemia in rats. Ann. Thorac. Surg. 105, 1523-1530.

Vilaça-Faria, H., Salgado, A.J., and Teixeira, F.G. (2019). Mesenchymal stem cells-derived exosomes: A new possible therapeutic strategy for Parkinson's disease? Cells 8, E118.

Wang, M., Yuan, Q., and Xie, L. (2019). Mesenchymal stem cell-based immunomodulation: Properties and clinical application. Stem Cells Int. 2018, 1-12.

Xiao, Z., Tang, F., Zhao, Y., Han, G., Yin, N., Li, X., Chen, B., Han, S., Jiang, X., Yun, C., Zhao, C., Cheng, S., Zhang, S., and Dai, J. (2018). Significant improvement of acute complete spinal cord injury patients diagnosed by a combined criteria implanted with NeuroRegen scaffolds and mesenchymal stem cells. Cell Transplant. 27, 907-915.

$\mathrm{Xu}, \mathrm{P}$., and Yang, X. (2019). The efficacy and safety of mesenchymal stem cell transplantation for spinal cord injury patients: A meta-analysis and systematic review. Cell Transplant. 28, 36-46.

Yousefifard, M., Nasseri Maleki, S., Askarian-Amiri, S.,Vaccaro, A.R, Chapman, J.R., Fehlings, M.G., Hosseini, M., and Rahimi-Movaghar, V. (2019). A combination of mesenchymal stem cells and scaffolds promotes motor functional recovery in spinal cord injury: A systematic review and metaanalysis. J. Neurosurg. Spine 1, 1-16.

Zhang, X.M., Ma, J., Sun, Y., Yu, B.Q., Jiao, Z.M., Wang, D., Yu, M.Y., Li, J.Y., and Fu, J. (2018). Tanshinone IIA promotes the differentiation of bone marrow mesenchymal stem cells into neuronal-like cells in a spinal cord injury model. J. Transl. Med. 16, 193.
Address correspondence to: Zijiang $Y u$ Department of Anatomy School of Basic Medical Sciences Guizhou Medical University Guiyang 550025 Guizhou China

E-mail: meimeibenben823@sina.com 